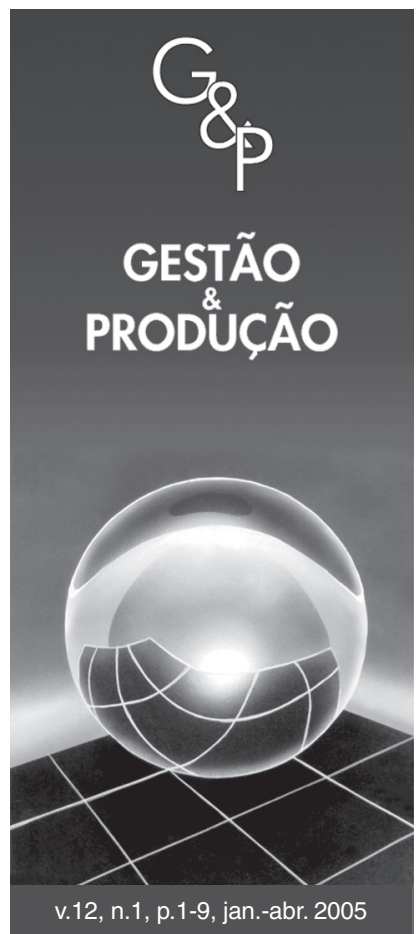

\title{
PROPOSTA PARA A GESTÃO DE ESTOQUES DE NOVOS PRODUTOS: SOLUÇÃO DO MODELO $(Q, r)$ PARA A DISTRIBUIÇÃO UNIFORME DA DEMANDA E DO LEAD-TIME DE SUPRIMENTO
}

\author{
Peter Wanke \\ Eduardo Saliby
}

Centro de Estudos em Logística, Instituto COPPEAD de Administração, Edifício do COPPEAD, Cidade Universitária, Ilha do Fundão, CEP 21949-900, Rio de Janeiro, RJ, e-mail: peter@ coppead.ufrj.br; saliby@ coppead.ufrj.br

Resumo

As premissas comumente consideradas nos modelos de estoques - aderência da demanda no lead-time de suprimento à Distribuição Normal, média e desvio-padrão conhecidos e lead-time de suprimento discreto - são freqüentemente irrealistas e podem implicar substanciais distorções na gestão dos estoques de novos produtos, sobretudo nos custos totais e nos indicadores de nível de serviço. Considerando que as empresas simultaneamente estocam os novos produtos e aprendem ao longo do tempo sobre as características da distribuição da demanda no lead-time de suprimento, é desenvolvida nesse artigo a solução do modelo $(Q, r)$ - tamanho de lote e ponto de pedido - para o caso da demanda e do lead-time de suprimento com Distribuição Uniforme. Por ser definida por meio de dois parâmetros mais intuitivos que a média e o desvio-padrão - máximo e mínimo - e representar situações em que qualquer resultado tem igual probabilidade de ocorrência, a premissa da Distribuição Uniforme pode constituir uma primeira abordagem prática para a gestão de estoques de novos produtos.

Palavras-chave: gestão de estoques, novos produtos, modelo $(Q, r)$, Distribuição Uniforme, análise de iniciativas gerenciais.

\section{Introdução}

A demanda e o lead-time de suprimento, bem como a demanda no lead-time de suprimento, podem não necessariamente ser aderentes à Distribuição Normal. Nesse sentido, diversos autores relatam o desenvolvimento de soluções analíticas e de heurísticas para a gestão de estoques sob condições de não-normalidade. Por exemplo, Jansen, Heus e Kok (1998) modelaram a demanda como um processo Bernoulli; Das (1976) desenvolveu uma solução aproximada para a Distribuição Gama da demanda no lead-time de suprimento, Hadley e Whitin (1961) analisaram o desempenho de diferentes modelos para a Distribuição de Poisson da demanda e a Distribuição Gama do lead-time de suprimento.

A aderência à Distribuição Normal pode ser uma premissa pouco realista para a gestão de estoques de produtos

no início do ciclo de vida. Além disto, quando um produto completamente novo é analisado, determinar suas distribuições da demanda, do lead-time de suprimento e da demanda no lead-time de suprimento com base em dados históricos ou no consumo histórico de produtos semelhantes pode simplesmente não ser viável ou factível. Alguns dados históricos devem ser coletados primeiro e só então análises podem ser conduzidas e conclusões podem ser tomadas. De fato, de acordo com Lariviere e Porteus (1999), "as empresas estão freqüentemente em dúvida sobre a real distribuição da demanda de um novo produto...as empresas simultaneamente estocam o produto e aprendem sobre a distribuição ao longo do tempo".

Especificamente, como as empresas podem lidar com a seguinte situação: produtos completamente novos, sem 
o registro de dados históricos, sem a possibilidade de criar analogias com produtos funcionalmente semelhantes e com a urgência de decidir tamanhos de lote e pontos de pedido? Como as empresas podem tomar decisões apropriadas com relação aos pontos de pedido, aos níveis de serviço e às faltas esperadas por ciclo de ressuprimento, se as distribuições da demanda e do lead-time de suprimento são ignoradas? Ou ainda, se sob a perspectiva de seus tomadores de decisão, quaisquer resultados da demanda e do lead-time de suprimento são igualmente prováveis, desde que compreendidos entre limites mínimos e máximos?

O objetivo deste artigo é propor um modelo de estoques que permita lidar de modo prático com estas questões. Considerando as propriedades da Distribuição Uniforme e as premissas comuns dos modelos de estoque como os pontos de partida, é desenvolvida a solução do modelo (Q,r) para a Distribuição Uniforme da demanda e do lead-time de suprimento, no caso relevante em que a demanda mínima e o lead-time de suprimento mínimo são iguais a zero. Em seguida, são apresentados um exemplo numérico e as conclusões do artigo.

\section{Premissas comuns dos modelos de estoque}

De acordo com Silver (1981), na maioria dos modelos de estoque diversas premissas são freqüentemente assumidas de modo implícito. Os efeitos em termos de custo e de nível de serviço decorrentes da assunção dessas premissas devem ser levados em consideração. Segundo o autor, as duas classes de premissas mais comuns nos modelos de estoque são (1) assumir uma determinada forma da distribuição da demanda, do lead-time de suprimento ou da demanda no lead-time de suprimento (quase sempre a Distribuição Normal) e (2) assumir que os parâmetros da distribuição são conhecidos, sobretudo sua média e seu desvio-padrão. Mentzer e Krishnan (1988) apontam uma terceira classe de premissas, freqüentemente aceita na literatura de operações e logística, para a determinação da variância $\left(\mathrm{s}_{\mathrm{x}}{ }^{2}\right)$ e do desvio-padrão $\left(\mathrm{s}_{\mathrm{x}}\right)$ da demanda do lead-time de suprimento: assumir uma distribuição contínua para a demanda, uma distribuição discreta para o lead-time de suprimento e a independência da demanda e do lead-time de suprimento. Esta premissa para a determinação da variância da demanda no lead-time de suprimento equivale, por exemplo, ao sorteio diário da demanda durante o lead-time de suprimento medido em dias, de modo que haja compensação das flutuações da demanda pelo efeito estatístico da soma de suas variâncias no lead-time de suprimento. Os autores demonstraram, por Transformação de Laplace, que sua expressão analítica é:

$$
\mathrm{s}_{\mathrm{X}}^{2}=\mathrm{T}^{*} \mathrm{~s}_{\mathrm{D}}^{2}+\mathrm{D}^{2 *} \mathrm{~s}_{\mathrm{T}}^{2}
$$

em que $\mathrm{T}=$ lead-time de suprimento esperado em dias, $\mathrm{s}_{\mathrm{D}}=$ desvio-padrão da demanda, $\mathrm{D}=$ demanda diária esperada e $\mathrm{s}_{\mathrm{T}}=$ desvio-padrão do lead-time de suprimento.

Com relação à primeira classe de premissas, já foi demonstrado que assumir a Distribuição Normal da demanda no lead-time de suprimento pode levar a significativas distorções na gestão de estoques, como, por exemplo, a determinação inadequada dos níveis de serviço, caso essa distribuição não seja de fato Normal. Mentzer e Krishnan (1988) investigaram o efeito da premissa da normalidade na determinação dos pontos de pedido para alcançar os níveis de serviço desejados. Os autores testaram duas diferentes distribuições para o lead-time de suprimento (Poisson e Geométrica) e três diferentes distribuições para a demanda (Exponencial, Normal e Gama), comparandoas com o caso da Distribuição Normal da demanda no lead-time de suprimento. Diferenças consideráveis foram relatadas com relação aos pontos de pedido e aos níveis de serviço alcançados por ciclo de ressuprimento, ilustrando os riscos associados a custos excessivos de manter estoques (elevados pontos de pedido) e a custos excessivos da falta de estoques (baixos pontos de pedido).

Lau (1989) apresentou um método para calcular pontos de pedido e níveis de serviço com base nos quatro momentos (média, variância, terceiro momento - assimetria e quarto momento - curtose) de uma dada distribuição da demanda no lead-time de suprimento. Com base em tabelas de referência para o percentil 95 da Distribuição Pearson padrão, disponível em textos avançados de estatística como o de Pearson e Hartley (1970), o autor demonstrou ser possível determinar um valor aproximado da quantidade de desvios-padrão da demanda no lead-time de suprimento $(\mathrm{k})$ com base na assimetria e na curtose da distribuição da demanda no lead-time de suprimento. $\mathrm{O}$ autor também apontou para os riscos de decisões equivocadas sobre pontos de pedido e níveis de serviço quando é considerada a Distribuição Normal da demanda no lead-time de suprimento.

Com relação à segunda classe de premissas, pode ser argumentado que a média e a variância da demanda no lead-time de suprimento podem ser facilmente calculadas a partir de dados históricos. Mas, se não houver dados históricos disponíveis, conforme mencionado na Introdução, como podem ser estimadas, do modo mais adequado, a média e a variância da demanda no leadtime de suprimento? Apesar de tomadores de decisão em algumas empresas conseguirem avaliar intuitivamente a magnitude da demanda média ou do lead-time de suprimento médio, de acordo com Lariviere e Porteus (1999), nem a variância, o desvio-padrão, a assimetria e a curtose são estatísticas fáceis de avaliar intuitivamente. As van- 
tagens de assumir a Distribuição Uniforme da demanda e do lead-time de suprimento, em casos como os novos produtos, estão relacionadas, sobretudo, à facilidade de incorporar avaliações subjetivas sobre seus valores máximos e mínimos.

Finalmente, com relação à terceira classe de premissas, autores como Brown (1982), Dell (1999), Christopher (2000) e Zipkin (2001) afirmam que em função do acirramento das pressões competitivas, o lead-time de suprimento das operações tende a se tornar uma variável de caráter contínuo e não mais de caráter discreto. Isso por que cada vez mais empresas clientes e fornecedoras estão organizando o ressuprimento dos estoques em "janelas de ciclo" ou em "janelas de serviço", caracterizadas por prazos de entrega contínuos, como, por exemplo, "de 24 até 72 horas", ou "em até 96 horas". O impacto da adoção de "janelas de ciclo" ou "janelas de serviço" equivale ao sorteio simultâneo da demanda diária e do lead-time de suprimento medido em dias. A variância do produto dessas duas variáveis aleatórias contínuas e independentes é dada por (Meyer, 1965):

$$
\mathrm{s}_{\mathrm{X}}^{2}=\mathrm{T}^{2 *} \mathrm{~s}_{\mathrm{D}}^{2}+\mathrm{D}^{2 *} \mathrm{~s}_{\mathrm{T}}^{2}+\mathrm{s}_{\mathrm{D}}{ }^{2 *} \mathrm{~s}_{\mathrm{T}}^{2}
$$

Nas próximas três seções são desenvolvidas as expressões analíticas para a função densidade de probabilidade, a média e a variância da distribuição da demanda no leadtime de suprimento, além das expressões analíticas para o nível de serviço e para a falta esperada por ciclo.

\section{Função densidade de probabilidade, média e variância da distribuição da demanda no tempo de reposta}

Sob a perspectiva do sorteio de valores aleatórios, a Distribuição Uniforme retorna valores compreendidos no intervalo delimitado entre um valor mínimo (m) e um valor máximo (M). Cada valor nesse intervalo possui a mesma densidade de probabilidade. Genericamente, pode-se afirmar que a Distribuição Uniforme pode ser empregada para denotar desconhecimento sobre os resultados de uma variável aleatória, exceto pelo fato desses resultados estarem compreendidos entre m e M (Meyer, 1965 e Ragsdale, 2000). Conforme apresentado nas seções anteriores, o ressuprimento dos estoques de novos produtos, quando não há dados históricos para avaliações preliminares das distribuições da demanda e do lead-time de suprimento, nem a possibilidade de estimá-las por analogias com outros produtos, poderia refletir essas premissas. Nesse caso, tanto as características da distribuição de probabilidade da demanda diária (D), quanto do lead-time de suprimento em dias $(\mathrm{T})$, seriam ignoradas.

De acordo com Meyer (1965), o produto da função densidade de probabilidade de duas variáveis aleatórias independentes é igual à função densidade de probabilida- de do produto dessas variáveis. Supondo D e T variáveis aleatórias contínuas, independentes e uniformemente distribuídas, sendo que $\mathrm{D}$ pode variar de $\mathrm{d}_{\mathrm{m}}$ (demanda diária mínima) a $\mathrm{d}_{\mathrm{M}}$ (demanda diária máxima) e $\mathrm{T}$ pode variar de $\mathrm{t}_{\mathrm{m}}$ (lead-time de suprimento mínimo em dias) a $\mathrm{t}_{\mathrm{M}}$ (lead-time de suprimento máximo em dias), a função densidade de probabilidade da demanda no lead-time de suprimento $(\mathrm{f}(\mathrm{x}))$ é dada por:

$$
\mathrm{f}(\mathrm{x})=1 /\left(\left(\mathrm{d}_{\mathrm{M}}-\mathrm{d}_{\mathrm{m}}\right) *\left(\mathrm{t}_{\mathrm{M}}-\mathrm{t}_{\mathrm{m}}\right)\right)
$$

Já demanda esperada no tempo de reposta (X) é o produto da demanda média diária pelo tempo médio de resposta em dias, ou seja:

$$
\mathrm{X}=\left(\left(\mathrm{d}_{\mathrm{M}}+\mathrm{d}_{\mathrm{m}}\right) *\left(\mathrm{t}_{\mathrm{M}}+\mathrm{t}_{\mathrm{m}}\right)\right) / 4
$$

Finalmente, a variância da demanda no lead-time de suprimento $\left(\mathrm{s}_{\mathrm{x}}^{2}\right)$ é dada por:

$$
\begin{aligned}
& \mathrm{s}_{\mathrm{X}}{ }^{2}=\left(\left(\mathrm{d}_{\mathrm{M}}-\mathrm{d}_{\mathrm{m}}\right)^{2 *}\left(\mathrm{t}_{\mathrm{M}}-\mathrm{t}_{\mathrm{m}}\right)^{2}+3 *\left(\mathrm{~d}_{\mathrm{M}}+\mathrm{d}_{\mathrm{m}}\right)^{2 *}\right. \\
& \left.\left(\mathrm{t}_{\mathrm{M}}-\mathrm{t}_{\mathrm{m}}\right)^{2}+3 *\left(\mathrm{~d}_{\mathrm{M}}-\mathrm{d}_{\mathrm{m}}\right)^{2 *}\left(\mathrm{t}_{\mathrm{M}}+\mathrm{t}_{\mathrm{m}}\right)^{2}\right) / 144
\end{aligned}
$$

\section{Nível de serviço por ciclo (CSL - Cycle Service Level)}

Segundo Glen, Lemis e Drew (2001), quando do cálculo do produto de duas variáveis aleatórias, é fundamental "considerar as magnitudes dos produtos das coordenadas dos vértices sudeste e noroeste do espaço delimitado pelas duas variáveis". Estes vértices definem três regiões de integração para a função densidade de probabilidade do produto de duas variáveis aleatórias uniformemente distribuídas. No caso do produto da demanda com o lead-time de suprimento, os produtos das coordenadas desses vértices são $t_{m} * d_{M}$ e $t_{M} * d_{m}$, e as regiões de integração também dependem do tamanho do ponto de pedido ( $\mathrm{r}$-reorder point). Na Figura 1 é ilustrado que dependendo do tamanho ponto do pedido, o nível de serviço por ciclo deve ser calculado em uma de três possíveis regiões de integração:

- Região de integração 1: $\mathrm{r}<=\mathrm{t}_{\mathrm{M}}{ }^{*} \mathrm{~d}_{\mathrm{m}}$;

- Região de integração 2: $\mathrm{r}>=\mathrm{t}_{\mathrm{m}}{ }^{*} \mathrm{~d}_{\mathrm{M}}$; e

- Região de integração 3: $\mathrm{t}_{\mathrm{M}}{ }^{*} \mathrm{~d}_{\mathrm{m}}<\mathrm{r}<\mathrm{t}_{\mathrm{m}} * \mathrm{~d}_{\mathrm{M}}$

De acordo com Silver e Peterson (1985), o nível de serviço por ciclo para $\mathrm{f}(\mathrm{x})$, em função de $\mathrm{r}$, é dado por:

$$
\operatorname{CSL}(r)={ }_{-\infty} \int \mathrm{r} f(x) d x
$$

Nas subseções seguintes são desenvolvidas as soluções analíticas do nível de serviço por ciclo para as três regiões de integração. Conforme é ilustrado na Figura 1, a função densidade de probabilidade da demanda no lead-time de suprimento está definida num simples espaço retangular, o que torna mais prática a integração dupla diretamente na demanda e no lead-time de suprimento em vez da Transformação Jacobiana (Meyer, 1965). 


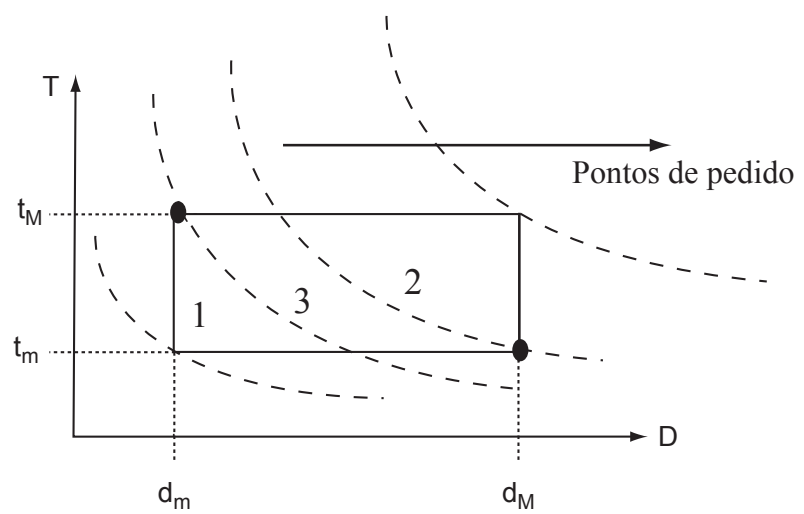

Figura 1. Regiões de Integração para f(x).

\section{Região de integração 1}

$$
\begin{aligned}
& \operatorname{CSL}(\mathrm{r})={ }_{\mathrm{dm}}{ }^{\mathrm{r} / \mathrm{m}}{ }_{\mathrm{tm}}{ }^{\mathrm{r} / \mathrm{d}} \mathrm{f}(\mathrm{x}) \mathrm{dt} d \mathrm{~d} \\
& \operatorname{CSL}(\mathrm{r})=\mathrm{f}(\mathrm{x}) *\left(\mathrm{r} * \ln \left(\mathrm{r} /\left(\mathrm{t}_{\mathrm{m}} * \mathrm{~d}_{\mathrm{m}}\right)\right)-\mathrm{r}+\mathrm{t}_{\mathrm{m}} * \mathrm{~d}_{\mathrm{m}}\right)
\end{aligned}
$$

\section{Região de integração 2}

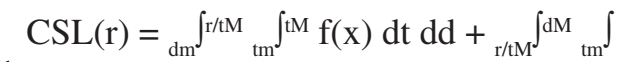

r/d $f(x) d t d d$

$$
\operatorname{CSL}(r)=f(x) *\left(\left(t_{\mathrm{M}}-t_{\mathrm{m}}\right) *\left(\mathrm{r} / \mathrm{t}_{\mathrm{M}}-\mathrm{d}_{\mathrm{m}}\right)+\right.
$$

$\left.\mathrm{r} * \ln \left(\left(\mathrm{d}_{\mathrm{M}} * \mathrm{t}_{\mathrm{M}}\right) / \mathrm{r}\right)-\left(\mathrm{t}_{\mathrm{m}} / \mathrm{t}_{\mathrm{M}}\right) *\left(\mathrm{~d}_{\mathrm{M}} * \mathrm{t}_{\mathrm{M}}-\mathrm{r}\right)\right)$

Se $\mathrm{d}_{\mathrm{m}}=0$ e $\mathrm{t}_{\mathrm{m}}=0$, a expressão (10) se converte no seguinte caso relevante para novos produtos:

$$
\operatorname{CSL}(\mathrm{r})=\mathrm{f}(\mathrm{x}) * \mathrm{r} *\left(1+\ln \left(\mathrm{d}_{\mathrm{M}} * \mathrm{t}_{\mathrm{M}} / \mathrm{r}\right)\right)
$$

\section{Região de integração 3}

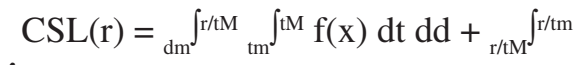

$\mathrm{tm}^{\mathrm{r} / \mathrm{d}} \mathrm{f}(\mathrm{x}) \mathrm{dt} d \mathrm{~d}$

$$
\begin{aligned}
& \operatorname{CSL}(\mathrm{r})=\mathrm{f}(\mathrm{x}) *\left(\left(\mathrm{t}_{\mathrm{M}}-\mathrm{t}_{\mathrm{m}}\right) *\left(\mathrm{r} / \mathrm{t}_{\mathrm{M}}-\mathrm{d}_{\mathrm{m}}\right)+\right. \\
& \left.\mathrm{r} * \ln \left(\mathrm{t}_{\mathrm{M}} / \mathrm{t}_{\mathrm{m}}\right)-\left(\mathrm{r} / \mathrm{t}_{\mathrm{M}}\right) *\left(\mathrm{t}_{\mathrm{M}}-\mathrm{t}_{\mathrm{m}}\right)\right)
\end{aligned}
$$

\section{Falta esperada por ciclo (ESC - Expec- ted Shortage per Cycle)}

Assim como no cálculo do nível de serviço por ciclo, o cálculo da falta esperada por ciclo deve observar as três regiões de integração ilustradas na Figura 1. Segundo Silver e Peterson (1985), a falta esperada por ciclo, em função de r, é dada por:

$$
\operatorname{ESC}(r)={ }_{\mathrm{r}}{ }^{+\infty} \mathrm{x} * \mathrm{f}(\mathrm{x}) \mathrm{dx}-\mathrm{r} *(1-\operatorname{CSL}(\mathrm{r}))
$$

\section{Região de integração 1}

$$
\begin{aligned}
& \operatorname{ESC}(r)={ }_{\mathrm{r} / \mathrm{tm}} \int \mathrm{dM}{ }_{\mathrm{tm}} \int \mathrm{tM} \mathrm{d}^{*} \mathrm{t}^{*} \mathrm{f}(\mathrm{x}) \mathrm{dt} \mathrm{dd}+{ }_{\mathrm{dm}} \mathrm{fr} / \mathrm{m} \\
& { }_{\mathrm{r} / \mathrm{d}}{ }^{\mathrm{tm}} \mathrm{d} \mathrm{d}^{*} \mathrm{t} \mathrm{f}(\mathrm{x}) \mathrm{dt} \mathrm{dd}-\mathrm{r} *(1-\operatorname{CSL}(\mathrm{r})) \\
& \operatorname{ESC}(r)=(f(x) / 4) *\left(t_{\mathrm{M}}{ }^{2}-\mathrm{t}_{\mathrm{m}}{ }^{2}\right) *\left(\mathrm{~d}_{\mathrm{M}}{ }^{2}-\mathrm{r}^{2} / \mathrm{t}_{\mathrm{m}}{ }^{2}\right)+
\end{aligned}
$$

$\mathrm{f}(\mathrm{x}) *\left(\left(\mathrm{t}_{\mathrm{M}}{ }^{2} / 4\right) *\left(\mathrm{r}^{2} / \mathrm{t}_{\mathrm{m}}{ }^{2}-\mathrm{d}_{\mathrm{m}}{ }^{2}\right)-\left(\mathrm{r}^{2} / 2\right) * \ln \left(\mathrm{r} /\left(\mathrm{t}_{\mathrm{m}} * \mathrm{~d}_{\mathrm{m}}\right)\right)\right)-\mathrm{r} *(1-$ CSL(r))

\section{Região de integração 2}

$$
\operatorname{ESC}(r)={ }_{\mathrm{r} / \mathrm{M}} \int_{\mathrm{r} / \mathrm{d}}^{\mathrm{dM}} \mathrm{fm}^{\mathrm{d}} \mathrm{d} * \mathrm{t} * \mathrm{f}(\mathrm{x}) \mathrm{dt} \mathrm{dd}-
$$

$\mathrm{r}^{*}(1-\operatorname{CSL}(\mathrm{r}))$

$$
\begin{gathered}
\operatorname{ESC}(\mathrm{r})=(\mathrm{f}(\mathrm{x}) / 2) *\left(\left(\mathrm{t}_{\mathrm{M}}{ }^{2} / 2\right) *\left(\mathrm{~d}_{\mathrm{M}}{ }^{2}-\mathrm{r}^{2} / \mathrm{t}_{\mathrm{M}}{ }^{2}\right)-\right. \\
\left.\mathrm{r}^{2} * \ln \left(\left(\mathrm{d}_{\mathrm{M}} * \mathrm{t}_{\mathrm{M}}\right) / \mathrm{r}\right)\right)-\mathrm{r} *(1-\operatorname{CSL}(\mathrm{r}))
\end{gathered}
$$

\section{Região de integração 3}

$$
\begin{aligned}
& \operatorname{ESC}(r)={ }_{\mathrm{r} / \mathrm{m}} \int \mathrm{dM}{ }_{\mathrm{tm}} \int \mathrm{tM} d * t * f(x) d t d d+ \\
& { }_{\mathrm{r} / \mathrm{M}} \int_{\mathrm{r} / \mathrm{d}}^{\mathrm{r} / \mathrm{d}} \mathrm{ftM}^{\mathrm{T}} \mathrm{d} * \mathrm{t}^{*} \mathrm{f}(\mathrm{x}) \mathrm{dt} \mathrm{dd}-\mathrm{r}^{*}(1-\operatorname{CSL}(\mathrm{r})) \\
& \mathrm{ESC}(\mathrm{r})=(\mathrm{f}(\mathrm{x}) / 4) *\left(\mathrm{t}_{\mathrm{M}}{ }^{2}-\mathrm{t}_{\mathrm{m}}{ }^{2}\right) * \\
& \left.\left(\mathrm{~d}_{\mathrm{M}}{ }^{2}-\mathrm{r}^{2} / \mathrm{t}_{\mathrm{m}}{ }^{2}\right)+(\mathrm{f}(\mathrm{x}) / 2) *\left(\mathrm{r}^{2} / 2\right) *\left(\left(\mathrm{t}_{\mathrm{M}}{ }^{2}-\mathrm{t}_{\mathrm{m}}{ }^{2}\right) / \mathrm{t}_{\mathrm{m}}{ }^{2}\right)-\mathrm{r}^{2} * \ln \left(\mathrm{t}_{\mathrm{M}} / \mathrm{t}_{\mathrm{m}}\right)\right) \\
& -\mathrm{r}^{*}(1-\operatorname{CSL}(\mathrm{r}))
\end{aligned}
$$

\section{Comparação dos resultados obtidos com a premissa da Distribuição Normal}

No artigo de Mentzer e Krishnan (1988), por exemplo, são apresentadas diversas situações nas quais resultados discrepantes em termos de ponto de pedido (e, conseqüentemente, em termos de nível de serviço por ciclo e de falta esperada por ciclo) são verificados quando se considera a Distribuição Normal da demanda no leadtime de suprimento para efeito de tomada de decisão, mas a real distribuição de probabilidade é outra (exponencial, gama e geométrica).

Resumidamente, esses resultados discrepantes podem ser classificados em dois tipos: no primeiro, o ponto de pedido deve ser menor do que aquele calculado com base na premissa da Distribuição Normal da demanda no leadtime de suprimento; no segundo tipo, o ponto de pedido deve ser maior do que aquele calculado com base nessa premissa. Discrepâncias do primeiro tipo implicam níveis de estoque desnecessários, com o oferecimento de níveis de serviço (CSL e ESC) acima das expectativas dos clientes. Já discrepâncias do segundo tipo implicam níveis de estoque aquém do necessário, sendo que os níveis de serviço desejados pelos clientes não são atingidos.

Para a maioria das situações identificadas por Mentzer e Krishnan (1988), a discrepância muda de direção para diferentes níveis de serviço ao cliente, quando são comparadas outras distribuições de probabilidade com a Distribuição Normal da demanda no lead-time de suprimento. O mesmo se verifica para a solução do caso da Distribuição Uniforme da demanda e do lead-time de suprimento. A mudança de direção das discrepâncias se deve aos diferentes formatos das funções de nível de serviço (CSL e ESC), conforme é ilustrado na Figura 2. 
De acordo com a Figura 2, para níveis de serviço por ciclo (CSL) entre 50 e $80 \%$, considerar a premissa da Distribuição Normal da demanda no lead-time de suprimento significa subestimar a probabilidade de não faltar produto, considerando-se a Distribuição Uniforme da demanda e do lead-time de suprimento como referência. Nessa região seriam verificadas discrepâncias do primeiro tipo, ou seja, planejar pela Distribuição Normal implicaria níveis de estoque desnecessários para atingir determinado nível de serviço por ciclo. Já para níveis de serviço por ciclo entre 80 e $98 \%$, considerar a Distribuição Normal significa superestimar a probabilidade de não faltar produto. Nessa região seriam verificadas discrepâncias do segundo tipo, ou seja, planejar pela Distribuição Normal implicaria níveis de estoque insuficientes para atingir determinado nível de serviço por ciclo. Para níveis de serviço próximos de $100 \%$ a discrepância é virtualmente inexistente.

Segundo Mentzer e Krishnan (1988), o único meio de evitar a armadilha causada por essas discrepâncias é realizar testes de aderência para determinar a real distribuição da demanda no lead-time de suprimento. Esta informação deve ser utilizada para serem calculados os respectivos parâmetros da real distribuição de probabilidade (por exemplo, no caso da Distribuição Normal, média e desviopadrão; no caso da Distribuição Gama, coeficientes alfa e beta; no caso da Distribuição Uniforme, máximo e mínimo), de modo a serem definidos corretamente os pontos de pedido para os diferentes níveis de serviço desejados.

Conforme apresentado nas seções 1 e 2 , o primeiro elemento complicador no caso de novos produtos é a inexistência de dados históricos sobre a demanda no leadtime de suprimento. Na impossibilidade de realizar testes de aderência, as empresas inicialmente estocam o produto e, ao longo do tempo, aprendem sobre a distribuição de probabilidade. Nessa fase inicial, mesmo que se suponha a premissa da Distribuição Normal, o segundo elemento complicador é como estimar, do modo mais adequado,

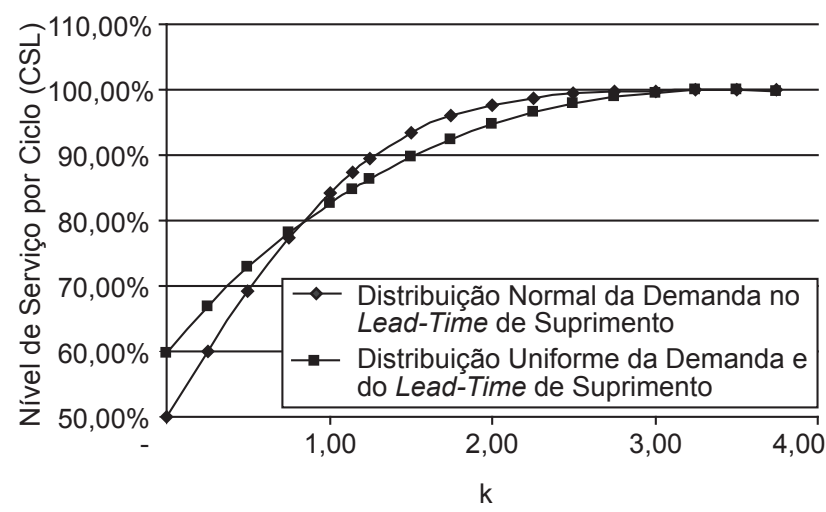

Figura 2. Discrepâncias no Nível de Serviço por Ciclo (CSL). a média e o desvio-padrão da demanda no lead-time de suprimento. Mesmo que seja possível estimar intuitivamente a magnitude da demanda média, nem a variância, o desvio-padrão, a assimetria e a curtose são estatísticas de fácil intuição. Tomando por base esses dois elementos complicadores, as vantagens de adotar a premissa da Distribuição Uniforme no caso de novos produtos estão relacionadas:

1. ao fato dessa distribuição ser reconhecidamente adequada para representar situações nas quais há completo desconhecimento sobre a real distribuição de probabilidades do evento em estudo; $\mathrm{e}$

2. a uma maior facilidade de estimar intuitivamente parâmetros como máximo e mínimo, comparativamente a parâmetros como desvio-padrão, curtose e assimetria.

Deve ser lembrado que Mentzer e Krishnan (1988) determinaram as discrepâncias por meio de confronto da premissa da Distribuição Normal com a identificação da real distribuição de probabilidade da demanda no lead-time de suprimento. No caso de novos produtos, identificar a real distribuição não é possível em função da inexistência de dados históricos. Nesse sentido, a Figura 2 constitui um indicativo da discrepância na gestão de estoques de novos produtos, quando o tomador de decisão adota a premissa da Distribuição Normal, mas sua única certeza é desconhecer a real distribuição de probabilidades da demanda no lead-time de suprimento e não ter meios para mensurá-la.

\section{Solução do modelo $(Q, r)$ para a região de integração 2}

Segundo Namit e Chen (1999), o modelo (Q,r) para o caso com faltas é dado por:

$$
\mathrm{TC}(\mathrm{Q}, \mathrm{r})=(\mathrm{Q} / 2+\mathrm{r}-\mathrm{X}) * \mathrm{~V} * \mathrm{C}+
$$$$
(\mathrm{D} / \mathrm{Q}) * 365 *(\mathrm{P}+\mathrm{S} * \mathrm{ESC}(\mathrm{r}))
$$

em que TC $=$ custo total por ano, $\mathrm{Q}=$ tamanho de lote, $\mathrm{r}$ = ponto de pedido, $\mathrm{X}=$ demanda esperada no lead-time de suprimento, $\mathrm{V}=$ custo por produto, $\mathrm{C}=$ taxa de oportunidade do capital por ano, $\mathrm{D}=$ demanda média diária, $\mathrm{P}=$ custo de colocação do pedido, $\mathrm{S}=$ custo da falta ou margem de contribuição, $\operatorname{ESC}(r)$ = falta esperada por ciclo de ressuprimento em função de $r$.

Considerando a situação relevante para um novo produto, na qual $\mathrm{d}_{\mathrm{m}}=0$ e $\mathrm{t}_{\mathrm{m}}=0$, ou seja, a região de integração 2, as expressões analíticas para $\mathrm{D}, \mathrm{s}_{\mathrm{X}}$ e r são, respectivamente, dadas por:

$$
\begin{aligned}
& \mathrm{D}=\mathrm{d}_{\mathrm{M}} / 2 \\
& \mathrm{~s}_{\mathrm{X}}=\sqrt{ } 7 * \mathrm{~d}_{\mathrm{M}} * \mathrm{t}_{\mathrm{M}} / 12 \\
& \mathrm{r}=\mathrm{X}+\mathrm{k} * \mathrm{~s}_{\mathrm{X}}
\end{aligned}
$$




$$
\mathrm{r}=\mathrm{d}_{\mathrm{M}} * \mathrm{t}_{\mathrm{M}} / 4+\mathrm{k} * \sqrt{ } 7 * \mathrm{~d}_{\mathrm{M}} * \mathrm{t}_{\mathrm{M}} / 12
$$

em que k é a quantidade de desvios-padrão da demanda.

Substituindo (25) em (11), a expressão do nível de serviço por ciclo em função da quantidade de desviospadrão da demanda é dada por:

$$
\operatorname{CSL}(\mathrm{k})=(1 / 4+\mathrm{k} * \sqrt{7} / 12) *(1+\ln (12 /(3+\mathrm{k} * \sqrt{ } 7)))
$$

Substituindo (25) e (26) em (18), a expressão da falta esperada por ciclo em função da quantidade de desviospadrão da demanda é dada por:

$$
\begin{gathered}
\operatorname{ESC}(\mathrm{k})=\mathrm{d}_{\mathrm{M}} * \mathrm{t}_{\mathrm{M}} *((1 / 4+\mathrm{k} * \sqrt{7 / 12}) \\
2 *(3 / 4+1 / 2 * \ln (12 /(3+\mathrm{k} * \sqrt{ } 7)))-\mathrm{k} * \sqrt{7 / 12})
\end{gathered}
$$

Finalmente, substituindo (22), (25) e (27) em (21), a expressão do modelo (Q,r) para o caso com faltas pode ser reescrita em função de $\mathrm{Q}$ e k:

$$
\begin{aligned}
& \mathrm{TC}(\mathrm{Q}, \mathrm{k})=\left(\mathrm{Q} / 2+\mathrm{k} * \sqrt{ } 7 * \mathrm{~d}_{\mathrm{M}} * \mathrm{t}_{\mathrm{M}} / 12\right) * \\
& \mathrm{~V} * \mathrm{C}+(365 / 2) *\left(\mathrm{~d}_{\mathrm{M}} / \mathrm{Q}\right) *\left(\mathrm{P}+\mathrm{S} * \mathrm{~d}_{\mathrm{M}} * \mathrm{t}_{\mathrm{M}} *\left((1 / 4+\mathrm{k} * \sqrt{7} / 12)^{2 *}\right.\right. \\
& *(3 / 4+1 / 2 * \ln (12 /(3+\mathrm{k} * \sqrt{7})))-\mathrm{k} * \sqrt{7} / 12)
\end{aligned}
$$

em que a primeira parcela da expressão (28) representa os custos de oportunidade de manter estoques de ciclo e de segurança e a segunda parcela, os custos de colocação do pedido e da falta por cada ciclo de ressuprimento.

Derivando (28) parcialmente em função de Q e k, igualando as derivadas a zero e expressando as derivadas em função de k, obtêm-se, respectivamente, as soluções para as quais o custo total é mínimo:

$$
\begin{aligned}
& \mathrm{Q}_{1}(\mathrm{k})=\left(\left(365^{*} \mathrm{~d}_{\mathrm{M}} *(\mathrm{P}+\mathrm{S} * \mathrm{ESC}(\mathrm{k})) /(\mathrm{V} * \mathrm{C})\right)^{1 / 2}\right. \\
& \mathrm{Q}_{2}(\mathrm{k})=\left(\left(-365 * \mathrm{~d}_{\mathrm{M}} * \mathrm{~S}\right) /(\mathrm{V} * \mathrm{C})\right)^{*}(\mathrm{k} * \sqrt{ } 7+(3+ \\
&\mathrm{k} * \sqrt{7}) * \ln (12 /(3+\mathrm{k} * \sqrt{ } 7))-9) / 24
\end{aligned}
$$

A expressão (29) é semelhante à fórmula do tamanho de lote econômico: no numerador são considerados os custos associados a cada ciclo de ressuprimento (colocação do pedido e falta) e no denominador o custo de oportunidade de manter estoques associado ao nível de estoque médio. Na expressão (30) são ponderados os custos da falta (numerador) e excesso (denominador). $\mathrm{O}$ custo total é mínimo para valores de $\mathrm{k}$ que satisfazem a igualdade $\mathrm{Q}_{1}(\mathrm{k})=\mathrm{Q}_{2}(\mathrm{k})$.

\section{Exemplo numérico e análises poten- ciais}

A demanda diária máxima de um novo produto é de 100 unidades e o lead-time de suprimento máximo é de 10 dias $\left(\mathrm{d}_{\mathrm{M}}=100\right.$ e $\left.\mathrm{t}_{\mathrm{M}}=10\right)$. Os valores mínimos da demanda e do lead-time de suprimento são iguais a zero $\left(\mathrm{d}_{\mathrm{m}}=0\right.$ e $\left.\mathrm{t}_{\mathrm{m}}=0\right)$. A taxa de oportunidade do capital é de $21 \%$ ao ano, o custo por produto é de $\$ 37,64$, o custo da falta por produto é de $\$ 2,85$ e o custo de colocação de cada pedido é de $\$ 148,21$. Para $\mathrm{k}=1,145$ (obtido por enumeração com o auxílio da planilha MS-Excel ${ }^{\circledR}$ ), o tamanho de lote é de aproximadamente de 999 unidades, o ponto de pedido é de 502 unidades, os custos totais são de aproximadamente $\$ 9.886,27$ por ano, o nível de serviço por ciclo é de $84,8 \%$ e a falta esperada por ciclo é de 23,77 unidades. Na Figura 3 é ilustrada a relação entre Q e k para este exemplo numérico.

Esse exemplo numérico ilustra não apenas como a gestão de estoques de um novo produto pode ser planejada por meio dos resultados desenvolvidos, mas também como diversas iniciativas para a gestão de cadeias de suprimento poderiam ter seus efeitos interpretados e quantificados à luz desses resultados. Por exemplo, é sabido que iniciativas como terceirização e parcerias tendem a reduzir os custos fixos de ressuprimento dos estoques (nesse caso, o parâmetro P). Menores custos fixos de ressuprimento implicam menores tamanhos de lote $(\mathrm{Q})$. O compartilhamento de informações no âmbito de iniciativas de gestão colaborativa, como o ECR (Efficient Consumer Response) e o CPFR (Collaborative Planning Forecasting \& Replenishment), permite que fabricantes de bens de consumo possam tomar decisões relativas à gestão de estoques com menor incerteza da demanda no lead-time de suprimento $\left(\mathrm{s}_{\mathrm{X}}\right)$.

\section{Validação dos resultados por simulação}

$\mathrm{O}$ modelo $(\mathrm{Q}, \mathrm{k})$ para os dados do exemplo numérico foi simulado em planilha MS-Excel, com o objetivo de validar as expressões analíticas para CSL (k), ESC (k) e TC (Q,k). Para cada par (Q,k) foram realizados 50 experimentos com 10.000 simulações uniperiodais para a demanda e o lead-time de suprimento uniformemente distribuídos. Na Tabela 1 é possível comparar os resultados teóricos derivados das soluções analíticas com os resultados empíricos obtidos por simulação. A margem de erro máxima observada foi de $0,75 \%$, e a média foi de $0,01 \%$. Os valores em negrito indicam a localização do

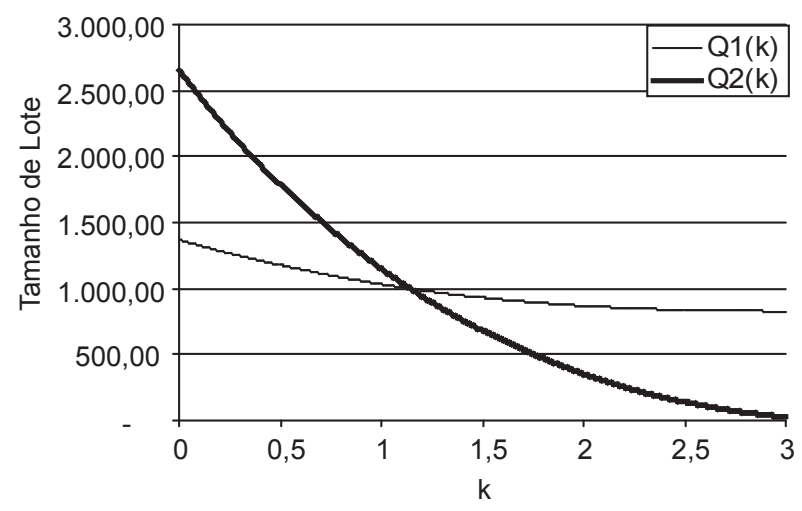

Figura 3. Relação entre Q e k para o Exemplo Numérico. 
mínimo teórico. Na Tabela 2 são apresentados os principais passos adotados para estruturar a simulação em planilha MS-Excel ${ }^{\circledR}$.

\section{Conclusões}

Neste artigo foi desenvolvida a solução analítica do modelo (Q,r) para a Distribuição Uniforme da demanda e do lead-time de suprimento. Essa solução foi validada por meio de simulações uniperiodais em planilha MS-Excel ${ }^{\circledR}$. Especificamente, foi analisado o caso relevante para o qual a demanda diária mínima e o lead-time de suprimento mínimo medido em dias são iguais a zero. Este caso relevante foi identificado a partir das três possíveis regiões de integração da função densidade de probabilidade.

A gestão de estoques de novos produtos representa um caso adequado à aplicação deste modelo, sobretudo em circunstâncias nas quais são desconhecidos as distri-

Tabela 1. Validação dos Resultados Teóricos por Simulação.

\begin{tabular}{|c|c|c|c|c|c|c|c|}
\hline & & \multicolumn{2}{|c|}{$Q=200$} & \multicolumn{2}{|c|}{$Q=400$} & \multicolumn{2}{|c|}{$Q=600$} \\
\hline & & Teórico & Simulado & Teórico & Simulado & Teórico & Simulado \\
\hline & CSL & $72,80 \%$ & $72,80 \%$ & $72,80 \%$ & $72,74 \%$ & $72,80 \%$ & $72,84 \%$ \\
\hline \multirow[t]{3}{*}{$\mathrm{k}=0,50$} & ESC & 53,34 & 53,27 & 53,34 & 53,56 & 53,34 & 53,20 \\
\hline & $\mathrm{TC}$ & 29057,14 & 29039,95 & 16149,92 & 16178,74 & 12374,47 & 12362,87 \\
\hline & CSL & $78,03 \%$ & $78,04 \%$ & $78,03 \%$ & $78,02 \%$ & $78,03 \%$ & $78,08 \%$ \\
\hline \multirow[t]{3}{*}{$\mathrm{k}=0,75$} & ESC & 39,82 & 39,76 & 39,82 & 39,85 & 39,82 & 39,73 \\
\hline & $\mathrm{TC}$ & 25978,31 & 25961,95 & 14828,35 & 14832,10 & 11638,66 & 11630,32 \\
\hline & CSL & $82,52 \%$ & $82,54 \%$ & $82,52 \%$ & $82,53 \%$ & $82,52 \%$ & $82,52 \%$ \\
\hline \multirow[t]{3}{*}{$\mathrm{k}=1,00$} & ESC & 28,98 & 28,85 & 28,98 & 28,79 & 28,98 & 29,00 \\
\hline & $\mathrm{TC}$ & 23594,85 & 23560,99 & 13854,46 & 13829,63 & 11134,63 & 11136,06 \\
\hline & CSL & $86,37 \%$ & $86,30 \%$ & $86,37 \%$ & $86,38 \%$ & $86,37 \%$ & $86,43 \%$ \\
\hline \multirow[t]{3}{*}{$\mathrm{k}=1,25$} & ESC & 20,44 & 20,57 & 20,44 & 20,38 & 20,44 & 20,27 \\
\hline & $\mathrm{TC}$ & 21808,05 & 21842,48 & 13178,91 & 13171,92 & 10829,49 & 10814,82 \\
\hline & CSL & $89,63 \%$ & $89,63 \%$ & $89,63 \%$ & $89,56 \%$ & $89,63 \%$ & $89,64 \%$ \\
\hline \multirow[t]{3}{*}{$\mathrm{k}=1,50$} & ESC & 13,85 & 13,87 & 13,85 & 13,85 & 13,85 & 13,80 \\
\hline & $\mathrm{TC}$ & 20530,20 & 20536,20 & 12757,83 & 12758,42 & 10694,00 & 10689,60 \\
\hline & CSL & $92,38 \%$ & $92,42 \%$ & $92,38 \%$ & $92,33 \%$ & $92,38 \%$ & $92,34 \%$ \\
\hline \multirow[t]{5}{*}{$\mathrm{k}=1,75$} & ESC & 8,91 & 8,89 & 8,91 & 8,98 & 8,91 & 8,96 \\
\hline & $\mathrm{TC}$ & 19682,36 & 19675,92 & 12551,75 & 12560,43 & 10701,84 & 10705,94 \\
\hline & & \multicolumn{2}{|c|}{$Q=800$} & \multicolumn{2}{|c|}{$Q=1000$} & \multicolumn{2}{|c|}{$Q=1200$} \\
\hline & & Teórico & Simulado & Teórico & Simulado & Teórico & Simulado \\
\hline & CSL & $72,80 \%$ & $72,85 \%$ & $72,80 \%$ & $72,82 \%$ & $72,80 \%$ & $72,71 \%$ \\
\hline \multirow[t]{3}{*}{$\mathrm{k}=0,50$} & ESC & 53,34 & 53,19 & 53,34 & 53,29 & 53,34 & 53,53 \\
\hline & $\mathrm{TC}$ & 10882,00 & 10872,13 & 10302,64 & 10300,06 & 10179,91 & 10188,361 \\
\hline & CSL & $78,03 \%$ & $78,01 \%$ & $78,03 \%$ & $77,86 \%$ & $78,03 \%$ & $78,02 \%$ \\
\hline \multirow[t]{3}{*}{$\mathrm{k}=0,75$} & ESC & 39,82 & 39,96 & 39,82 & 40,06 & 39,82 & 39,82 \\
\hline & $\mathrm{TC}$ & 10439,00 & 10447,82 & 10035,43 & 10047,96 & 10029,84 & 10029,56 \\
\hline & CSL & $82,52 \%$ & $82,48 \%$ & $82,52 \%$ & $82,44 \%$ & $82,52 \%$ & $82,48 \%$ \\
\hline \multirow[t]{3}{*}{$\mathrm{k}=1,00$} & ESC & 28,98 & 29,05 & 28,98 & 29,05 & 28,98 & 29,01 \\
\hline & $\mathrm{TC}$ & 10169,90 & 10174,11 & 9907,29 & 9910,84 & 9995,67 & 9996,82 \\
\hline & CSL & $86,37 \%$ & $86,38 \%$ & $86,37 \%$ & $86,35 \%$ & $86,37 \%$ & $86,36 \%$ \\
\hline \multirow[t]{3}{*}{$\mathrm{k}=1,25$} & ESC & 20,44 & 20,38 & 20,44 & 20,43 & 20,44 & 20,48 \\
\hline & $\mathrm{TC}$ & 10050,00 & 10046,43 & 9898,48 & 9898,14 & 10060,95 & 10062,827 \\
\hline & CSL & $89,63 \%$ & $89,63 \%$ & $89,63 \%$ & $89,72 \%$ & $89,63 \%$ & $89,60 \%$ \\
\hline \multirow[t]{3}{*}{$\mathrm{k}=1,50$} & ESC & 13,85 & 13,83 & 13,85 & 13,70 & 13,85 & 13,84 \\
\hline & $\mathrm{TC}$ & 10057,30 & 10056,25 & 9991,46 & 9983,64 & 10211,05 & 10210,542 \\
\hline & CSL & $92,38 \%$ & $92,32 \%$ & $92,38 \%$ & $92,34 \%$ & $92,38 \%$ & $92,36 \%$ \\
\hline \multirow[t]{2}{*}{$\mathrm{k}=1,75$} & ESC & 8,91 & 8,96 & 8,91 & 8,91 & 8,91 & 8,93 \\
\hline & $\mathrm{TC}$ & 10172,10 & 10175,24 & 10170,44 & 10170,43 & 10432,81 & 10433,513 \\
\hline
\end{tabular}


Tabela 2. Passos da Simulação Realizada em MS-Excel ${ }^{\circledR}$.

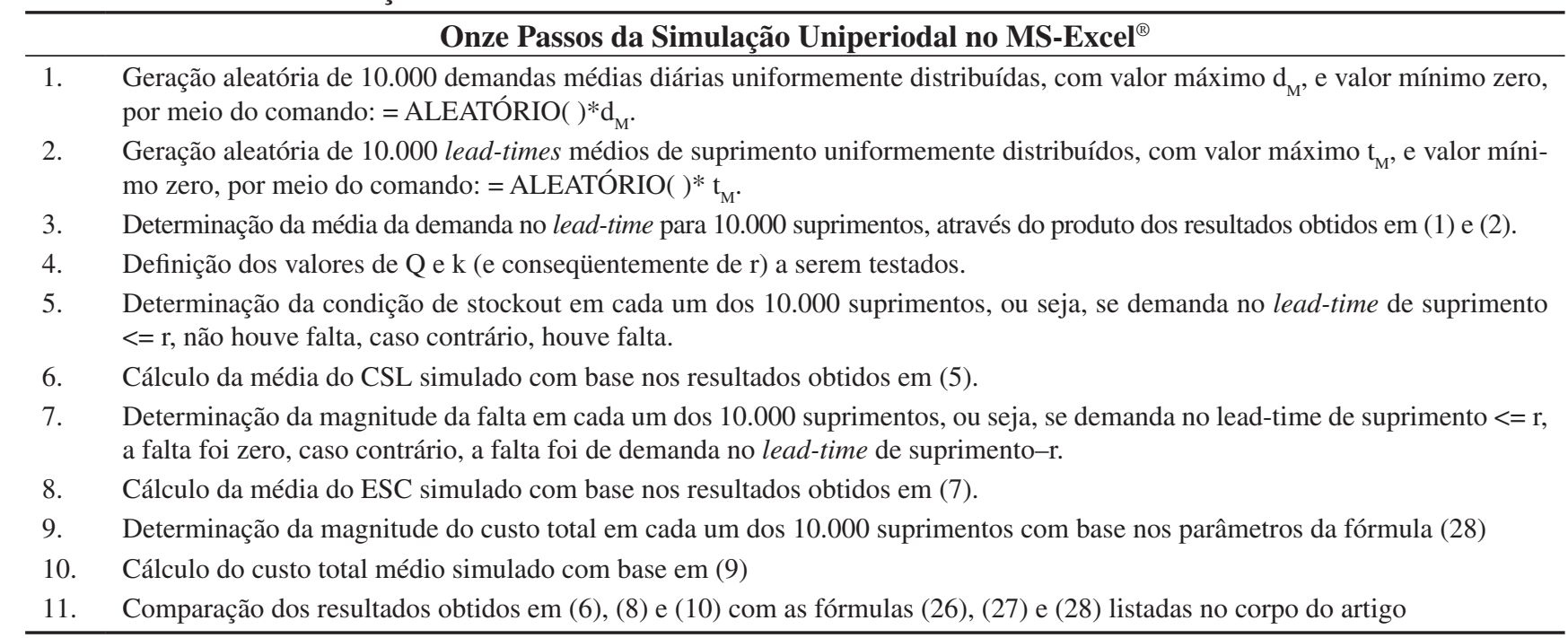

buições de probabilidade da demanda e do lead-time de suprimento e os seus principais parâmetros. Avaliações subjetivas sobre os valores máximo e mínimo da demanda e do lead-time de suprimento podem ser facilmente incorporadas nesse modelo, conforme ilustrado pelo exemplo numérico. Tomadores de decisão em empresas podem utilizar esses resultados, facilmente implementáveis em planilha MS-Excel ${ }^{\circledR}$.

Os resultados desenvolvidos também podem ser empregados para interpretar e analisar o impacto de iniciativas para a gestão de cadeias de suprimentos nos níveis de estoque de novos produtos. Basicamente porque iniciativas como parcerias, terceirização e ressuprimento contínuo implicam redução nos custos fixos de ressuprimento e na variabilidade da demanda no lead-time de suprimento.
Finalmente, pesquisas e artigos futuros sobre a aplicação de diferentes distribuições de probabilidade na gestão de estoques deveriam considerar a Distribuição Triangular, definida pelos parâmetros média, mínimo e máximo. Diferentemente da Distribuição Uniforme, a premissa da Distribuição Triangular da demanda e do lead-time de suprimento poderia ser empregada em diferentes estágios do ciclo de vida do produto, e não apenas no estágio de introdução para novos produtos. Caberia ao tomador de decisão avaliar intuitivamente valores mínimos, máximos e médios para poder planejar com base nos resultados teóricos para CSL, ESC e TC. No entanto, o desenvolvimento dessas soluções analíticas passaria pela análise de 6 regiões de integração, caracterizando a solução para a premissa da Distribuição Triangular como um problema mais complexo que o da Distribuição Uniforme.

\section{Referências Bibliográficas}

BROWN, R.G. Advanced service parts inventory control. Vermont: Materials Management Systems Inc., 1982.

CHRISTOPHER, $M$. The agile supply chain - competing in volatile markets. Industrial Marketing Management, v. 29 , n. 1 , p. $37-44,2000$.

DELL, M. Direct from dell. New York: Harperbusiness, 1999.

DAS, C. Approximate solution to the (Q,r) inventory model for gamma lead-time demand. Management Science, v. 22 , n. 9, p. 1043-1047, 1976.

GLEN, A.; LEEMIS, L.M.; DREW, J.H. Computing the distribution of the product of two continuous random variables. Working paper NY 10996. Department of Mathematical Sciences, The United States Military Academy. West Point, 2001.

HADLEY, G.; WHITIN, M. A family of inventory models. Management Science, v. 7, n. 4, p. 351-371, 1961.

JANSEN, F; HEUTS, R.; KOK, T. On the (R,s,Q) inventory model when demand is modeled as a compound bernoulli process. European Journal of Operational Research, n. 104, p. 423-436, 1998.

LARIVIERE, M.; PORTEUS, E. Stalking information: bayesian inventory management with unobserved lost sales. Management Science, v. 45, n. 3, p. 346-363, 1999. 
LAU, H. Toward an inventory control system under nonnormal demand and lead-time uncertainty. Journal of Business Logistics, v. 10, n. 1, p. 88-103, 1989.

MENTZER, J.T.; KRISHNAN, R. The effect of the assumption of normality on inventory control/customer Service. Journal of Business Logistics, v. 6, n. 1, p. 101-120, 1988.

MEYER, P. Introductory probability and statistical applications. New York: Addison-Wesley Publishing Company, 1965.

NAMIT, K.; CHEN, J. Solutions to the (Q,r) inventory model for gamma lead-time demand. International Journal of Physical Distribution \& Logistics Management, v. 29, n. 2, p. 138-151, 1999.
PEARSON, E.; HARTLEY, H. Biometrika tables for statisticians. Cambridge: Cambridge University Press, 1970.

RAGSDALE, C. Spreadsheet modeling and decision analysis. New Jersey: South-Western Thomson Learning, 2000.

SILVER, E. Operations research in inventory management: a review and critique. Operations Research, v. 29, n. 4, p. 628-645, 1981.

SILVER, E.; PETERSON, R. Decision systems for inventory management and production planning. New York: Wiley \& Sons, 1985.

ZIPKIN, P. The limits of mass customization. MIT Sloan Management Review, v. 42, n. 3, p. 81-87, 2001.

\section{A PROPOSAL FOR NEW PRODUCT INVENTORY MANAGEMENT: SOLUTION OF THE (Q,r) INVENTORY MODEL FOR UNIFORM DISTRIBUTION OF DEMAND AND LEAD-TIME}

\section{Abstract}

The premises commonly adopted in inventory management models - adherence of lead-time demand to Normal Distribution, known average and standard deviations, and discrete lead time - are often unrealistic and can lead to considerable distortions in new product inventories, particularly insofar as total costs and service level indicators are concerned. Considering that companies stock new products while simultaneously learning about the characteristics of lead-time demand distribution, this paper proposes the solution to the $(Q, r)$ - order quantity and reorder point - inventory model for uniform demand and lead-time. The premise of Uniform Distribution is defined by two parameters that are more intuitive than mean and standard deviation - maximum and minimum - and it can also be applied when a result shows the same probability of occurring. Therefore, its adoption may be the first practical approach for new product inventory management.

Keywords: inventory management, new products, $(Q, r)$ inventory model, Uniform Distribution, analysis of management initiatives. 